\title{
POLITECNICO
}

\section{MILANO 1863}

\section{RE.PUBLIC@POLIMI}

Research Publications at Politecnico di Milano

Waveguide arrays for light harvesting in microfluidic chips

Surya S. Guduru; Petra Paiè; Serena Bolis; Roberto Osellame; Roberta Ramponi;

Tersilla Virgili; Krishna C. Vishnubhatla

Published in:

Optical Engineering, 53(7), 071811 (2014).

Copyright 2014 Society of Photo-Optical Instrumentation Engineers (SPIE). One print or electronic copy may be made for personal use only. Systematic reproduction and distribution, duplication of any material in this paper for a fee or for commercial purposes, or modification of the content of the paper are prohibited.

When citing this work, cite the original published paper.

Surya S. Guduru, Petra Paiè, Serena Bolis, Roberto Osellame, Roberta Ramponi, Tersilla Virgili, and Krishna C. Vishnubhatla "Waveguide arrays for light harvesting in microfluidic chips," Optical Engineering 53(7), 071811 (4 February 2014).

The final publication is available at: https://doi.org/10.1117/1.0E.53.7.071811

Permanent link to this version

http://hdl.handle.net/11311/939358 


\section{Optical Engineering}

\section{Waveguide arrays for light harvesting in microfluidic chips}

Surya. S. K. Guduru

Petra Paiè

Serena Bolis

Roberto Osellame

Roberta Ramponi

Tersilla Virgili

Krishna Chaitanya Vishnubhatla 


\title{
Waveguide arrays for light harvesting in microfluidic chips
}

\author{
Surya. S. K. Guduru, ${ }^{a, b}$ Petra Paiè, ${ }^{b}$ Serena Bolis, ${ }^{b}$ Roberto Osellame, ${ }^{c}$ Roberta Ramponi, ${ }^{a, b, c}$ Tersilla Virgili, ${ }^{c, *}$ and \\ Krishna Chaitanya Vishnubhatla ${ }^{a}$ \\ aIstituto Italiano di Tecnologia, Center for Nano Science and Technology @ PoliMi, Via Pascoli 70/3, Milan 20133, Italy

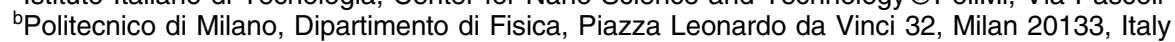 \\ 'Istituto di Fotonica e Nanotecnologie-CNR, Piazza Leonardo da Vinci 32, Milan 20133, Italy
}

\begin{abstract}
We demonstrate a way of light harvesting in integrated microfluidic chips fabricated by femtosecond laser micromachining. The architecture consists of waveguide arrays fabricated in the vicinity of the microchannel filled with a fluorescent organic solution (e.g., polyfluorene solution). Amplified spontaneous emission from the microchannel is efficiently coupled by the waveguides to the outside of the chip. ๑ 2014 Society of Photo-Optical Instrumentation Engineers (SPIE) [DOI: 10.1117/1.OE.53.7.071811]
\end{abstract}

Keywords: waveguide arrays; femtosecond laser; micromachining; light harvesting.

Paper 131655SS received Oct. 31, 2013; revised manuscript received Dec. 19, 2013; accepted for publication Dec. 20, 2013; published online Feb. 4, 2014.

\section{Introduction}

The field of optofluidics involves the combination of microfluidic and optical functionalities in developing versatile lab-on-chip devices. It incorporates the advantages of both microfluidics and optics. Lab-on-chip devices with various functionalities offer a good platform for biological applications like biosensors, cell sorters, etc. Optofluidics exploits the properties of fluids for transporting purposes which are otherwise absent in solid state devices. In addition to these, the low material consumption and the ability to tune and reconfigure the device by replacement of the fluids are great advantages. ${ }^{1-3}$ Further, such optofluidics can be integrated with other micro-optical elements with ease giving rise to "Lab-on-a-chip" devices.

Fused silica offers a great platform for biological applications due to its transparency and most importantly, due to fact that it is highly inert, easily available, and moldable into desired shapes. It also has other good properties like non porosity, durability, etc. ${ }^{4,5}$ Previously, techniques borrowed from the semiconductor electronics industry like photolithography, were employed in order to create microstructures in glass. These techniques, involving masking steps time consuming, are inefficient for prototyping. Femtosecond laser micromachining is a relatively new technique that has become a reliable fast prototyping tool for realizing novel microfluidic devices, due to its versatility for fabricating complex optofluidic networks and circuits. It can also be employed as a tool in surface as well as bulk micromachining in transparent materials. The advantage of this technique is also that the fabrication process is inherently three-dimensional (3-D) in nature, and it is cost effective as no masks are needed. ${ }^{6-9}$ The other main advantage lies in the fabrication of optical structures like waveguides and the imprint for microfluidic channels in the same step, simplifying the integration and alignment processes. ${ }^{10}$

Tight focusing of femtosecond-laser pulses leads to very high intensities of the order of $\approx 10 \mathrm{TW} / \mathrm{cm}^{2}$ inside glass, causing nonlinear processes like multiphoton absorption. This localized deposition of energy induces permanent structural and material damages, leading to the modification of refractive index of the irradiated region. Femtosecondlaser irradiation followed by chemical etching (FLICE) is the technique employed in fabricating the optofluidic channels. ${ }^{11-14}$ The substrate after irradiation is etched in an aqueous solution of hydrogen fluoride (HF) acid to create micro channels. Direct writing of waveguides inside glass is also accomplished by femtosecond-laser writing but at much smaller intensities, leading to a gentle refractive index modification. ${ }^{15}$ Thus, femtosecond-laser micromachining is a useful tool for the realization of real 3-D structures fabricated directly in the bulk glass and the single fabrication step of both waveguides and micro channels simplifies the proper alignment.

Femtosecond laser-fabricated waveguide arrays have been applied in light harvesting and manipulation previously. Thomson et al. ${ }^{16}$ proposed efficient collection of light from the telescope by multimode optical fibers and 3-D photonic devices, the latter were fabricated by Ultrafast Laser Inscription. Corrielli et al. ${ }^{17}$ have used waveguide arrays in creating photonic lattices by femtosecond-laser micromachining to observe quantum analogy of fractional Bloch oscillations.

In this work, we report the application of such arrays in light harvesting in an optofluidic scenario. An integrated chip with waveguides and micro channel was previously demonstrated by Osellame et al. ${ }^{10}$ where single waveguides were fabricated in a direction perpendicular to the micro channel. In this work, we demonstrate a novel way of light harvesting in integrated microfluidic chips fabricated by femtosecond-laser micromachining where the light emitted by the active material placed inside the microchannel gets coupled into the waveguide array and then gets collected at the end face of it. Light harvesting from micro channels by waveguides becomes an important issue in many applications 
concerning transportation of light from one part of the chip to the other. Applications where multiple light sources and light wavelength conversion are needed on the same chip require light to be transported from one part of the device to the other. The architecture consists of waveguide arrays fabricated in the vicinity of the end face of the microchannel. These micro channels are filled with a fluorescent organic solution of poly (9,9-dioctylfluorene, PFO) in decahydronaphthalene (decalin) with $15 \mathrm{gL}^{-1}$ concentration. An optical excitation with sufficient energy density above the amplified spontaneous emission (ASE) threshold of the polymer is sent on the microchannel. The array of waveguides collects and guides the ASE emission, which otherwise would diverges.

\section{Experimental}

Figure 1 shows the schematic of experimental setup for the fabrication of microchannels and waveguide array. A femtosecond-laser capable of delivering pulses of 400-fs, at $1040 \mathrm{~nm}$ and with $960-\mathrm{kHz}$ repetition rate was used for the fabrication. The infrared light delivered by the laser was converted into the second harmonic $(520 \mathrm{~nm})$ by a beta barium borate crystal. Microchannels in the chip were fabricated by the FLICE technique. In the first step, the fused silica substrate was translated by means of an $X Y Z$-translation stage. In order to avoid tapering of the microchannels during etching, a conical shape was irradiated [Fig. 2(a)] such that after etching, we obtain a very uniform microchannel with circular cross sections with a diameter ranging between 120 and $150 \mu \mathrm{m}$. In this case, the cross-section diameter of the microchannel is $145 \mu \mathrm{m}$.

The fabrication parameters of microchannels and waveguides were optimized (Table 1). The one step fabrication ensures a good alignment between the micro channel and
Table 1 The parameters for the waveguide writing and the microchannel imprint.

\begin{tabular}{|c|c|c|c|c|}
\hline Type & $\begin{array}{l}\text { Energy per } \\
\text { pulse (nJ) }\end{array}$ & $\begin{array}{l}\text { Laser } \\
\text { repetition } \\
\text { rate }(\mathrm{kHz})\end{array}$ & $\begin{array}{c}\text { Sample } \\
\text { speed } \\
(\mathrm{mm} / \mathrm{s})\end{array}$ & Microscope \\
\hline Waveguide & 300 & 20 & 10 & $20 \times, 0.45 \mathrm{NA}$ \\
\hline $\begin{array}{l}\text { Micro } \\
\text { channel }\end{array}$ & $\approx 500$ & 480 & 1 & $50 \times, 0.5 \mathrm{NA}$ \\
\hline
\end{tabular}

the waveguide array. The process consists first of irradiation of the sample with the femtosecond-laser to fabricate waveguides and to create the imprint of the channel [Fig. 2(a)]; then the irradiated chip is etched in an ultrasonic bath with $20 \%$ aqueous solution of $\mathrm{HF}$ acid for $3 \mathrm{~h}$ at $35^{\circ} \mathrm{C}$, to create the hollow microchannel. Figure 2(b) shows the picture of the microchannel after etching with the waveguide array on the vicinity. Figure 3(a) shows the image of the cross section of the waveguide array on the polished edge of the chip, obtained using a standard optical microscope (4× magnification).

\section{Discussion}

The waveguide array consists of a $15 \times 15$ matrix of waveguides in the shape of a diamond [Fig. 3(a)], and the picture is obtained using a standard optical microscope $4 \times$ magnification. The waveguides are separated by a distance of about $12 \mu \mathrm{m}$. A single waveguide fabricated with the same parameters as above is characterized for its loss, the refractive index contrast, the numerical aperture, and the mode profile. By standard loss characterization, the propagation loss is

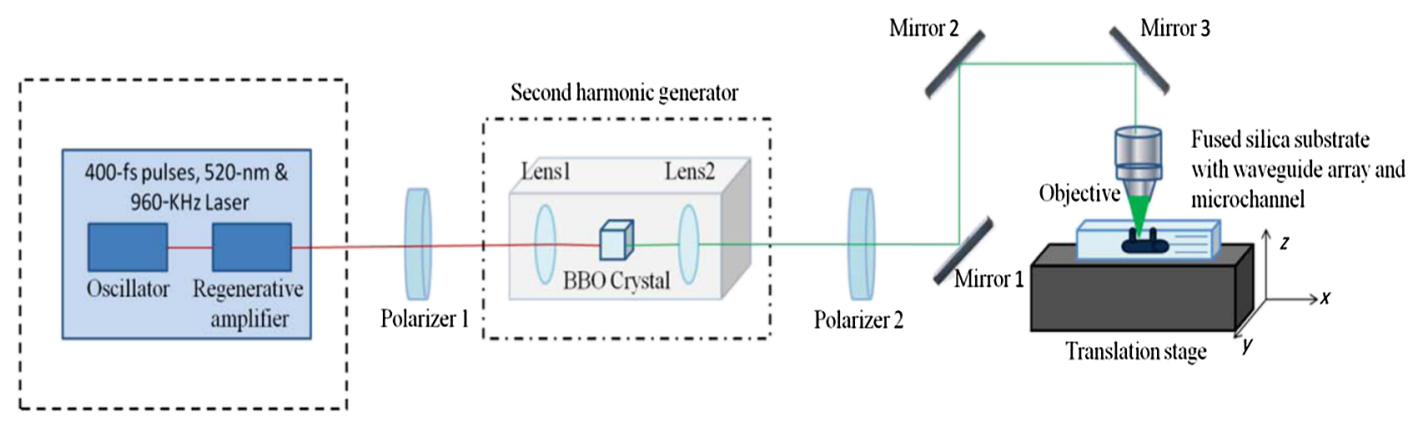

Fig. 1 The schematic diagram of the setup for the integrated chip fabrication.

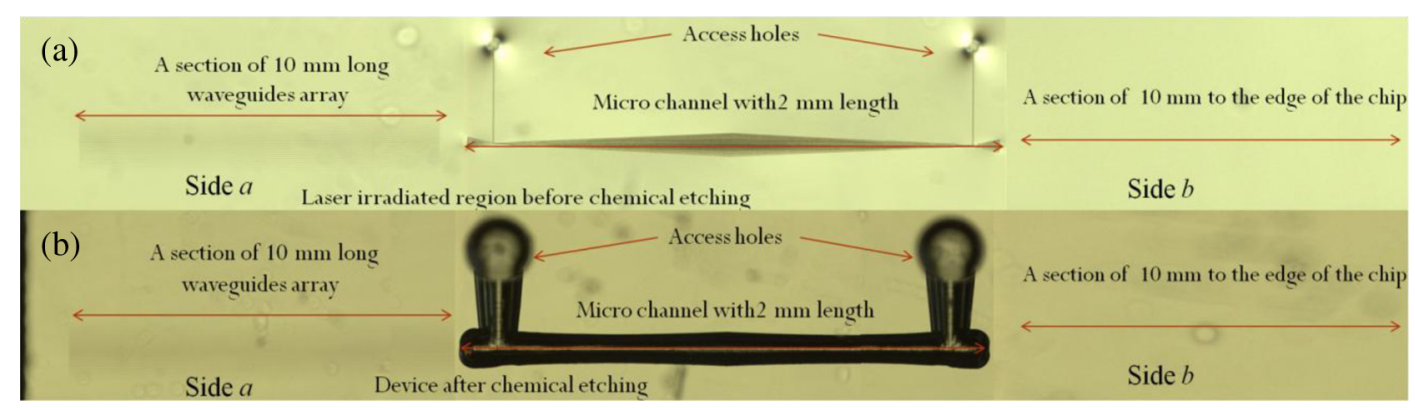

Fig. 2 (a) Femtosecond-laser irradiated region and (b) microchannel after etching, with the waveguides bunch in the vicinity. 

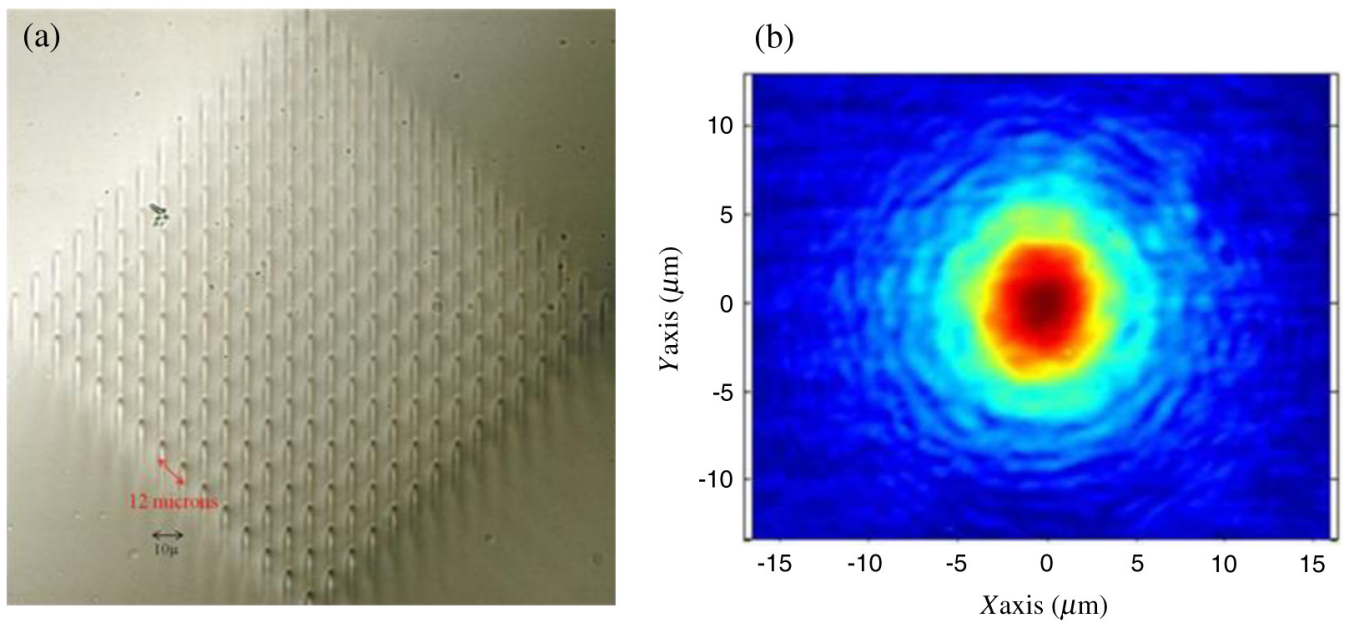

Fig. 3 The waveguide array (optical microscope) (a) and the mode profile of a single waveguide (CCD camera) (b).

evaluated to be $<1 \mathrm{~dB} / \mathrm{cm}$ at $630 \mathrm{~nm}$, the source being a He-Ne laser. The waveguides have a slightly elliptical cross section, but the mode field dimensions were found to be around $20 \mu \mathrm{m}$ in both $x$ and $y$ [Fig. 3(b)].

Following the technique in Ref. 18, we found a refractive index contrast $\Delta n \approx 7 \mathrm{E}-4$ and a numerical aperture, $\mathrm{NA} \approx 0.045$. These are typical values for waveguides fabricated by femtosecond-laser micromachining. In Fig. 4, we report the schematic diagram to demonstrate the light harvesting by the array of waveguides, and we placed an optical filter to remove the laser line excitation, and the image of the waveguide array cross section was obtained using a $20 x$ objective on the CCD camera (Hamamatsu Infrared Vidicon, Hamamatsu City, Japan). Waveguide arrays with different lengths $(2.5,5$, and $10 \mathrm{~mm})$ were fabricated and tested for harvesting. In this work, we report specifically the results for the 10 -mm waveguide arrays.

The PFO solution inside the micro channel was excited by the second harmonic of a Ti:sapphire femtosecond laser source $\left(\lambda_{\text {exc }}=400 \mathrm{~nm}\right.$, pulse time duration of $\left.60 \mathrm{fs}\right){ }^{12}$ The stripe excitation $(30 \mu \mathrm{m} \times 2 \mathrm{~mm})$ was created using a cylindrical lens in order to excite the entire length of the microchannel. The effective refractive index of the PFO solution $(\approx 1.6)$ is higher than the surrounding glass medium, hence the channel can act as a waveguide. Therefore, the
ASE from the solution inside the microchannel is mostly guided along the channel axis. After the end face of the channel, it is further guided by the waveguides array until the edge of the chip. The ASE from the waveguide array is collected by a fiber coupled to an optical multichannel analyzer (OMA, Princeton Instruments Acton SP2150, 300 gr/nm, New Jersey). In the absence of the waveguide array, the ASE signal would have been lost due to divergence.

Figure 5(a) shows the image of the waveguide cross section at the edge of the chip imaged by the CCD camera when the PFO solution inside the microchannel was under excitation. It clearly resembles the shape of the waveguide cross section in Fig. 3(a). Figure 5(b) shows the intensity of the light guided by the waveguides array. It can be noticed in the images obtained by the CCD camera that only waveguides toward the bottom corner guide a larger intensity of the ASE [Figs. 5(a) and 5(b)]. This can be due to the presence of a slight offset of the micro channel with respect to the waveguides bunch (i.e., the waveguides array cross section and the micro channel cross section were not perfectly aligned, since the micro channel cross section was smaller than the waveguide array cross section) and to the fact that the most of the excitation light does not reach the entire volume of the solution in the channel but it is absorbed in the first $50 \mu \mathrm{m}$ of the solution.

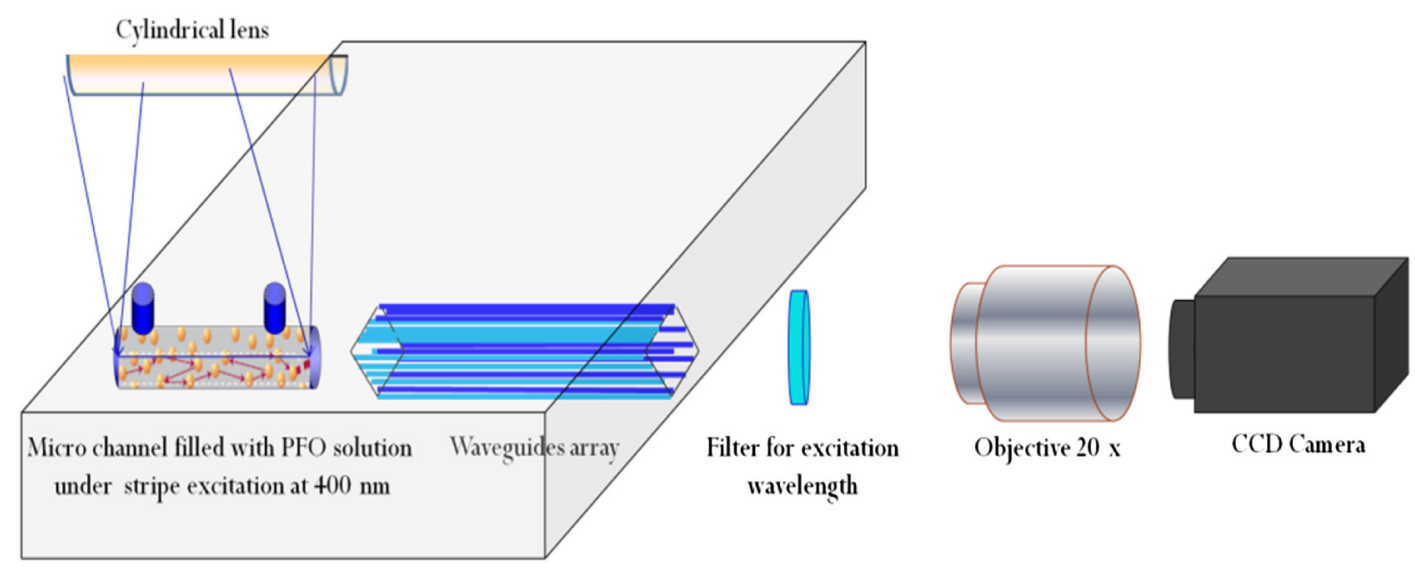

Fig. 4 Schematic experimental setup for the light collection. 

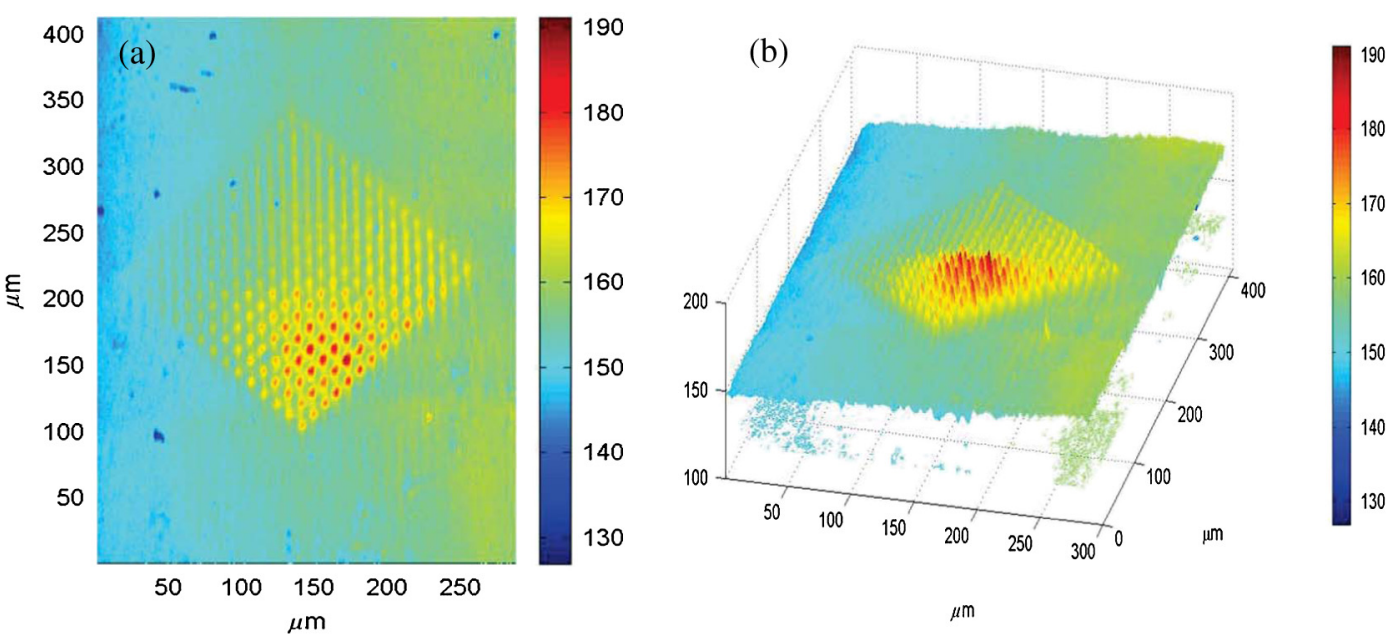

Fig. 5 The image of the cross section of waveguide array coupling the amplified spontaneous emission (ASE) to the edge of the chip (a) and the three-dimensional intensity of the ASE guided to the edge of the chip by the waveguides array (b).

Figure 6 shows images from the CCD camera obtained at the same excitation conditions on the end faces of a second chip where the channel is placed at the center of it with a waveguide array on one side $a$ [Fig. 6(a)] and just fused silica on the other side $b$ [Fig. 6(b)]. From side $a$, it is possible to see the light being guided by the waveguides bunch, while from side $b$ the light emitted is scattered away and diverges so the intensity detected seems to be almost zero using the same intensity scale for the two images [see Fig. 6(b)]. Actually from both sides, it is possible to detect the emission.
Figure 6(c) shows the spatial integrated photoluminescence (PL) intensity spectrum obtained using a collecting fiber bundle with overall $\approx 1$-mm-core diameter connected to the OMA. The overall spatial integrated PL intensity detected is similar from both sides, so this indicates that we have been able to confine, and guide all the ASE emission into the waveguide array, and so to deliver it to the external edge of the chip.

We have thus successfully demonstrated light harvesting by waveguides array in an optofluidic chip. This leads us to
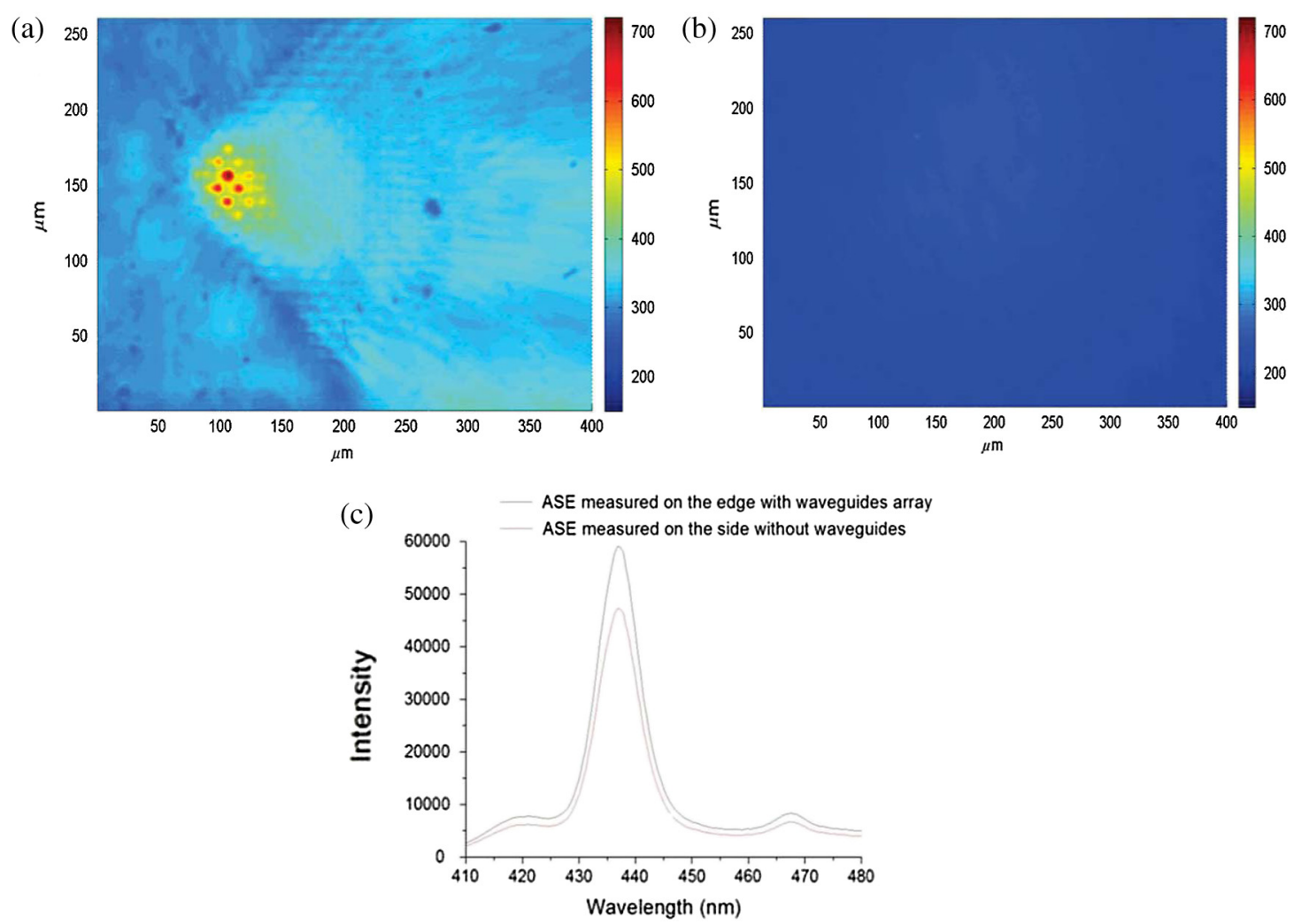

Fig. 6 (a) Image of the edge of the chip with waveguide array showing light getting coupled. (b) Image of the edge of the chip without the waveguide bunch under the same excitation condition. (c) The ASE measured on the side of the waveguides and on the side without the waveguides. 
novel architectures like multiple sources on single chip or guiding light from one part of the chip to the other. Increasing the refractive index contrast and thus the NA of the waveguides, it is possible to improve the efficiency of light harvesting, which is proposed as a future assignment.

\section{References}

1. C. Monat, P. Domachuk, and B. J. Eggleton, "Integrated optofluidics: a new river of light," Nature Photon. 1(2), 106-114 (2007).

2. L. Pang et al., "Optofluidic devices and applications in photonics, sensing and imaging," Lab Chip 12(19), 3543-3551 (2012).

3. Y. Hanada, K. Sugioka, and K. Midorikawa, "Highly sensitive optofluidic chips for biochemical liquid assay fabricated by 3D femtosecond laser micromachining followed by polymer coating," Lab Chip 12(19), 3688-3693 (2012).

4. J. F. Dishinger and R. T. Kennedy, "Serial immunoassays in parallel on a microfluidic chip for monitoring hormone secretion from living cells," Anal. Chem. 79(3), 947-954 (2007).

5. P. Watts, "Microreactors for drug discovery: the importance of integrating chemical synthesis with real-time analytical detection," Anal. Bioanal. Chem. 382(4), 865-867 (2005).

6. Y. Bellouard et al., "Fabrication of high-aspect ratio, micro-fluidic channels and tunnels using femtosecond laser pulses and chemical etching," Opt. Express 12(10), 2120-2129 (2004).

7. V. Maselli et al., "Fabrication of long microchannels with circular cross section using astigmatically shaped femtosecond laser pulses and chemical etching," Appl. Phys. Lett. 88(19) 191107 (2006).

8. F. Serra et al., "Topological defects of nematic liquid crystals confined in porous networks," Soft Matter 7(22), 10945-10950 (2011).

9. K. C. Vishnubhatla et al., "Femtosecond laser direct writing of gratings and waveguides in high quantum efficiency erbium-doped Baccarat glass," J. Phys. D: Appl. Phys. 42(20), 205106 (2009).

10. R. Osellame et al., "Integration of optical waveguides and microfluidic channels both fabricated by femtosecond laser irradiation." Appl. Phys. Lett. 90(23), 231118 (2007).

11. K. C. Vishnubhatla et al., "Effect of configuration of the microchannels fabricated by femtosecond laser micromachining on topological defects in confined liquid crystals," Proc. SPIE 8249, 82490B (2012).

12. K. C. Vishnubhatla et al., "Femtosecond laser fabrication of microfluidic channels for organic photonic devices," Appl. Opt. 48(31), G114-G118 (2009).

13. K. C. Vishnubhatla et al., "Ultrafast optofluidic gain switch based on conjugated polymer in femtosecond laser fabricated micro channels," Appl. Phys. Lett. 94(4), 041123 (2009).

14. T. Virgili et al., "Ultrafast optical gain switch in organic photonic devices," J. Mater. Chem. 20(3), 519-523 (2010).

15. R. Osellame, G. Cerullo, and R. Ramponi, Femtosecond Laser Micromachining: Photonic and Microfluidic Devices in Transparent Materials, Springer, Berlin, Heidelberg (2012).

16. R. R. Thomson et al., "Ultrafast laser inscription: an enabling technology for astrophotonics," Opt. Express 17(3), 1963-1969 (2009).

17. G. Corrielli et al., "Fractional Bloch oscillations in photonic lattices," Nat. Commun. 4, 1-6 (2013).

18. D. Homoelle et al., "Infrared photosensitivity in silica glasses exposed to femtosecond laser pulses,"Opt. Lett. 24(18), 1311-1313 (1999).

Surya S. K. Guduru received his MSc (photonics specialization) degree in physics from Sri Satya Sai Institute of Higher Learning, Andhrapradesh, India, in 2011. He is currently a PhD student at the Center for Nanoscience and Technology, Milano, Italy, working with the femtosecond laser micromachining group. His area of research is "light harvesting structures fabricated by femtosecond laser micromachining in integrated optofluidic applications."

Petra Paiè received her MSc in physics engineering in 2011 at Politecnico of Milano, Italy, and is currently in the second year of $\mathrm{PhD}$ at the Department of Physics of the same university. Her main research activity deals with femtosecond laser micromachining for the fabrication of optofluidic devices, in particular concerning biological applications.

Serena Bolis graduated in physical engineering in 2012 at Université Libre de Bruxelles, Belgium (specialization photonics) and in 2013 at Politecnico di Milano, Italy (specialization nanotechnology) in a double degree program. Since 2013 she has carried out a joint PhD between Universiteit Gent and Université Libre de Bruxelles, Belgium. Her research activity is about soliton propagation in dye-doped nematic liquid crystals.

Roberto Osellame received his laurea degree in electronic engineering from the Politecnico di Milano (Italy) in 1996 and the PhD degree in physics from the Politecnico di Torino (Italy) in 2000. Since 2001 he is a contract professor at Politecnico di Milano. Since 2007 he has been a senior researcher at the Institute for Photonics and Nanotechnologies (IFN) of the Italian National Research Council (CNR). His research interests include integrated devices and femtosecond laser micromachining of transparent material.

Roberta Ramponi is the director of the CNR Institute of Photonics and Nanotechnology and full professor of physics at the Politecnico di Milano. Her research activity includes integrated and nonlinear optics, photonic circuits for quantum optics, and microoptofluidic devices for biomedical and environmental sensing. She has been the president of the European Optical Society in 2006 to 2008 and is now a member of the Executive Committee of the European Technology Platform Photonics21.

Tersilla Virgili graduated in physics at University of Bologna, Italy, in 1996. In 2000 she received a PhD in physics from the University of Sheffield, UK. Since 2001 she is a permanent researcher at the Institute of Photonics and Nanotechnologies (IFN) of the CNR of Italy and contract professor at Politecnico di Milano. Her research activity involves different research fields such as condensed matter physics, ultrafast spectroscopy, organic microcavities, realization, and characterization of organic devices.

Krishna Chaitanya Vishnubhatla received his $\mathrm{PhD}$ degree in physics co-tutored by the Department of Physics, University of Hyderabad, India, and CNR-IFN, Dipartimento di Fisica University of Trento, Italy, in 2008. He worked as a senior postdoctoral researcher at CNST-IIT @ polimi (2010 to 2011). From 2012 to 2013 he worked as a research technologist, team leader for femtosecond laser micromachining group at CNST. Currently he is an assistant professor, Department of Physics, Sri Satya Sai Institute of Higher Learning, Andhrapradesh, India. 